\title{
Spontaneous Bleedings in COVID-19 Patients: An Emerging Complication
}

\author{
Irene Bargellini ${ }^{1}\left[\right.$ Rosa Cervelli $^{1} \cdot$ Alessandro Lunardi $^{1} \cdot$ Rossella Scandiffio $^{1}$. \\ Francesco Daviddi $^{1} \cdot$ Luigi Giorgi $^{1} \cdot$ Antonio Cicorelli $^{1} \cdot$ Laura Crocetti $^{1}$. \\ Roberto Cioni ${ }^{1}$
}

Received: 8 April 2020/Accepted: 25 April 2020/Published online: 17 May 2020

(C) Springer Science+Business Media, LLC, part of Springer Nature and the Cardiovascular and Interventional Radiological Society of Europe (CIRSE) 2020

To the Editor,

We read with great interest the "Joint CIRSE-APSCVIR checklist to prepare IR departments for COVID 19," recently available on the official site of CIRSE society [1], that provides a useful checklist to guarantee the healthcare professionals' safety and to prevent the spread of the infection, focusing on interventional radiology (IR) departments.

In the complex clinical scenario of COVID-19 patients, several IR procedures could be necessary to manage fluid collections, abscesses, acute thromboembolisms and hemorrhages caused by traumas or gastrointestinal complications.

Due to the growing data about the association between coronavirus infection and coagulopathy, anticoagulant therapy with low molecular weight heparin (LMWH) has become part of the medical therapy in hospitalized patients, to reduce the risk of venous thromboembolism [2]. This approach, however, may increase the risks of spontaneous bleeding, especially in elderly patients with comorbidities.

Over the past 4 days, four consecutive COVID-19 patients with spontaneous bleedings underwent endovascular embolization in our center. The first case was a 74-year-old woman with large left pectoral muscle hematoma due to rupture of superior thoracic artery, probably occurring during patient's mobilization while she was on the respirator in the prone position. The second case was 71-year-old male with large hematoma of left iliopsoas

Irene Bargellini

irenebargellini@hotmail.com

1 Department of Interventional Radiology, Pisa University Hospital, Via Paradisa 2, 56126 Pisa, Italy muscle; the patient required electrical cardioversion 2 days before due to atrial fibrillation, possibly causing traumatic injury of lumbar arteries [3]. On the 3rd day, we observed a large rectus sheath hematoma extending into the prevesical space of Retzius fed by both the right and left inferior epigastric arteries, in a 71-year-old woman with renal insufficiency. All three cases were prescribed 6000UI LMWH/12 h. The last case was a 81-year-old man with a large hematoma of the left thigh fed by multiple branches of the profunda femoral artery, who was prescribed a unusual high dose of LMWH (8000UI/12 h). All cases were successfully managed by coil embolization.

We have organized our IR workflow for COVID-19 patients, paying particular attention to the planning of dedicated workflows and protocols, staff training, preparation of the angiosuite before the patient's arrival, and accurate staff decontamination and cleaning of the rooms after the procedure. In the specific setting of hemorrhage, we realized the importance of having blood products already available in the angiosuite even in hemodynamically stable patients, in case blood transfusion is needed during the procedure. Moreover, while planning the dedicated workflows, it is important to take into account the role of the anesthesiologist. In the specific case of a COVID-19 patient, the anesthesiologist accompanies the patient from the intensive care unit to the angiographic suite. This condition implies that he/she is to be considered "contaminated" when entering the angiosuite. To ensure radiation protection, dedicated aprons and thyroid shields should made be available to be worn by the anesthesiologist above the potentially contaminated personal protective equipments, before entering the angiosuite. They would then be decontaminated at the end of the procedure. 
In conclusion, in the complex clinical scenario of COVID-19 patients, we should take into account also a possible risk of coagulopathy and spontaneous bleeding, which would require active involvement of IRs in an emergency setting. Prophylaxis with LMWH has been recommended in these patients, although it should be administered with caution, taking into account comorbidities, possible disease-related thrombocytopenia and potential interactions with other drugs. IR units must therefore be prepared to manage bleedings, by setting specific workflows and paying attention to any potential source of contamination.

\section{Compliance with Ethical Standards}

Conflict of interest The authors declare that they have no conflict of interest.

\section{References}

1. Checklist for preparing your IR service for COVID-19. https:// www.cirse.org/wp-content/uploads/2020/04/cirse_APSCVIR_ Checklist_COVID19_prod.pdf.

2. Clinical management of severe acute respiratory infection (SARI) when COVID-19 disease is suspected-Interim guidance 13 March 2020. World Health Organization. https://www.who.int/ docs/default-source/coronaviruse/clinical-management-of-novelcov.pdf.

3. Jahollari A, Cavolli R, Tavlasoglu M, Sallahu F, Muriqi S. Iliopsoas hematoma due to muscular rupture following defibrillation. Ulus Travma Acil Cerrahi Derg. 2013;19(6):564-6.

Publisher's Note Springer Nature remains neutral with regard to jurisdictional claims in published maps and institutional affiliations. 